\title{
APORTACIONES AL CONOCIMIENTO DE LOS LÓBULOS DE SOLIFLUXIÓN Y CAMBIOS AMBIENTALES HOLOCENOS EN SIERRA NEVADA
}

\author{
MARC OLIVA $^{1}$ \\ LOTHAR SCHULTE ${ }^{1}$ \\ Antonio Gómez OrTiz ${ }^{1}$
}

\begin{abstract}
Resumen - Las cabeceras de los valles de San Juan y Río Seco (Sierra Nevada) acogen un notable desarrollo de geoformas solifuidales, entre ellas los lóbulos. El control térmico y dinámico de uno de éstos, en el circo de Río Seco, muestra que la solifluxión permanece inactiva, a pesar de existir una capa helada estacional de hasta $70 \mathrm{~cm}$ de profundidad. Respecto a la génesis de los lóbulos de solifluxión, el estudio sedimentológico permite deducir fases de actividad solifluidal alternándose con fases de desarrollo edáfico durante el Holoceno tardío.
\end{abstract}

Palabras clave: Control térmico, lóbulos de solifluxión, monitorización dinámica, Holoceno, Sierra Nevada.

\begin{abstract}
CONTRIBUTION TO THE UNDERSTANDING OF SOLIFLUCTION LOBES activity and Holocene environmental changes in Sierra Nevada (Southern SPAIN): Headwaters of San Juan and Rio Seco valleys (Sierra Nevada) concentrate an important number of solifuction features. Thermal and dynamic monitoring of a solifluction lobe in the Rio Seco cirque show that despite a seasonal frozen layer of $70 \mathrm{~cm}$ thickness, solifluction processes remain inactive. Sedimentological studies of different solifluction lobes show phases of enhanced solifluction activity during the Late Holocene alternated with periods of edaphic development.
\end{abstract}

Key words: Geomorphological monitoring, thermal monitoring, solifluction lobes, Holocene, Sierra Nevada.

Resumo - CONTRIBUTOS PARA O CONHECIMENTO DOS LÓBULOS DE SOLIFLUXÃO E

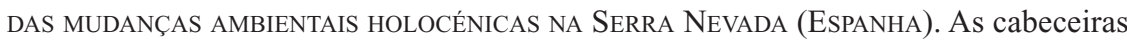
dos vales de San Juan e do Rio Seco, na Serra Nevada, conservam um grande número de formas de solifluxão, entre as quais estão os lóbulos. Os registos térmicos e dinâmicos no circo glaciário do rio Seco mostram que a solifluxão não está activa, apesar de haver uma superfície gelada estacional, que pode atingir os $70 \mathrm{~cm}$ de espessura.

Recebido: 24/11/2007. Revisto: 07/04/2009. Aceite: 19/05/2009.

1 Departamento de Geografía Física y Análisis Geográfico Regional y Servicio de Gestión y Evolución del Paisaje. Universidad de Barcelona. E-mail: oliva_marc@yahoo.com 
O estudo sedimentológico de vários lóbulos mostra que houve fases actividade de solifluxão alternando com períodos de desenvolvimento edáfico, durante o Holocénico tardio.

Palavras-chave: Controle térmico, lóbulos de solifluxão, monitorização dinâmica, Holocénico, serra Nevada.

Résumé - Quelques donnéES SuR LeS LOBES DE SOlifluXION ET LES CHANGEmENTS ENVIRONNEMENTAUX holocènes DANS la SierRa NEVAda (ESPAGNe). La tête des vallées de San Juan et de Rio Seco est le siège d'un notable développement de formes solifluidales et, en particulier, de lobes. La surveillance thermique et dynamique de l'un d'eux, dans le cirque de Rio Seco, a montré que la solifluxion n'est pas actuellement active, malgré l'existence d'une couche gelée saisonnière qui atteint 70 $\mathrm{cm}$ de profondeur. En ce qui concerne la genèse des lobes de solifluxion, l'étude sédimentologique permet de montrer que des phases d'activité solifluidale ont alterné avec des phases de développement édaphique, pendant l'Holocène tardif.

Mots clés: Contrôle thermique, lobes de solifluxion, surveillance dynamique, Holocène, Sierra Nevada.

\section{INTRODUCCIÓN}

La solifluxión se define como el lento movimiento de la capa superficial del suelo pendiente abajo, por efecto de procesos del hielo-deshielo en el mismo suelo (Ballantyne y Harris, 1994). Tradicionalmente los estudios sobre solifluxión se han centrado en áreas donde su acción afecta directamente a infraestructuras y equipamientos, es decir, altas latitudes y montañas elevadas de latitudes medias. En la península Ibérica, por la marginalidad de los sectores afectados por procesos fríos periglaciares, su estudio, y en particular, el relativo a la inestabilidad del suelo, se ha venido focalizando en un reducido número de enclaves montañosos. Entre ellos, recientemente caben destacarse Pirineo Central (Chueca y Julián, 1995), Sistema Central (Palacios et al., 2003), Serra da Estrela (Vieira, 2004) y Sierra Nevada (Gómez Ortiz et al., 2006).

El objetivo del presente artículo es profundizar en la génesis de la solifluxión en Sierra Nevada y para ello se caracterizarán las formas resultantes, se determinará su dinamismo actual y se propondrá una cronología de los procesos desencadenantes durante el Holoceno tardío.

\section{CARACTERÍSTICAS DEL ÁMBITO DE ESTUDIO}

A $37^{\circ}$ de latitud Norte y $3^{\circ}$ de longitud Oeste se extiende con orientación W-E el macizo de Sierra Nevada, con alturas que superan holgadamente los 3000 metros (Mulhacén 3478 m, Veleta 3398 m), las más altas de la Península Ibérica. Su posición geográfica, a caballo del cinturón de altas presiones subtropicales y los vientos del oeste de latitudes medias, singulariza el interés por 
estudiar los procesos geomorfológicos fríos que el macizo acoge, particularmente por los acusados gradientes verticales que genera la Sierra, de tanto interés en el comportamiento de los diferentes parámetros climáticos en montañas de latitudes medias (Wanner et al., 2004).

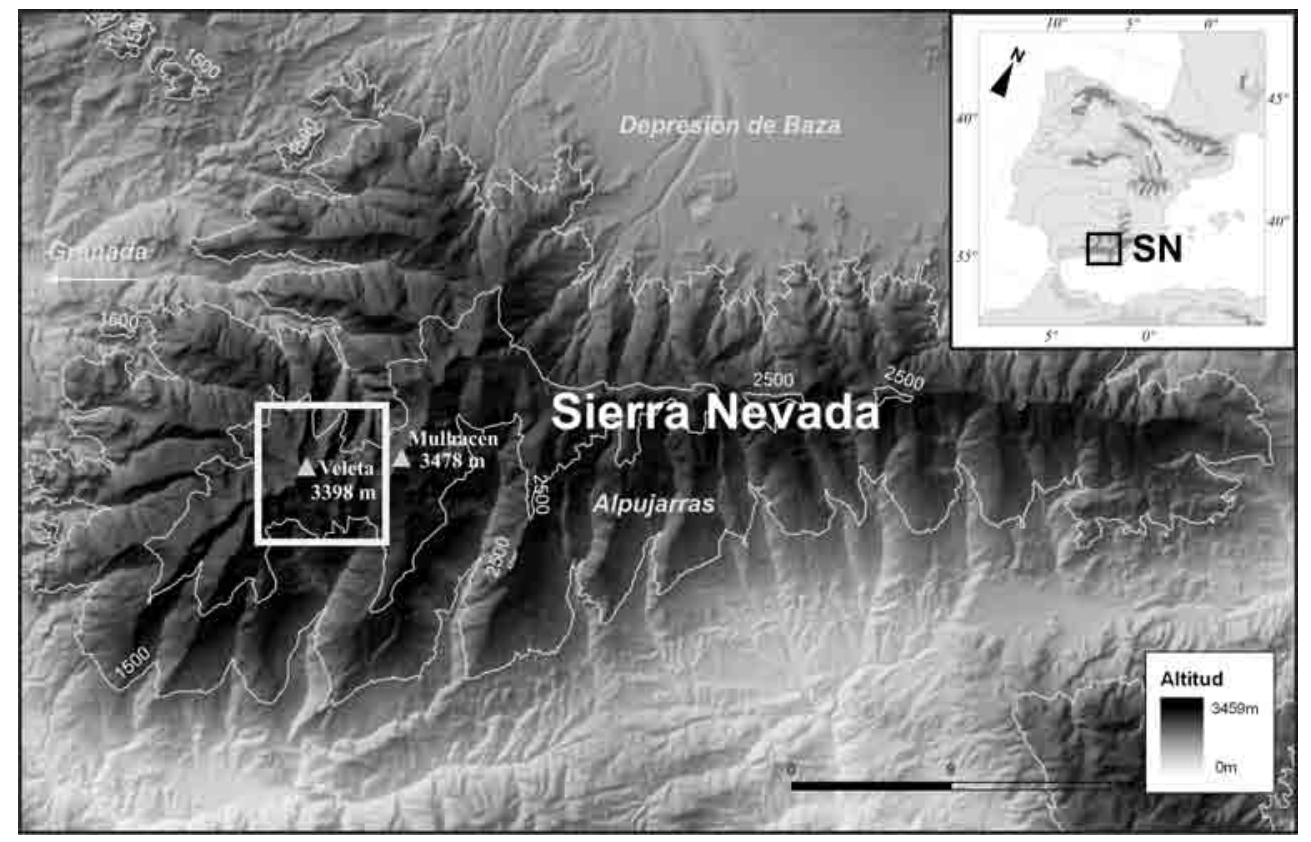

Fig. 1 - Localización del área de estudio en Sierra Nevada.

Fig. 1 - Location of the study area in Sierra Nevada.

Sierra Nevada es un macizo de marcada aridez, lo que explica su escasa cobertura vegetal, sobre todo en cumbre, aunque incluye un elevado número de endemismos (Molero Mesa y Pérez Raya, 1987). La litología dominante en sus tramos elevados se caracteriza por la presencia de micasquistos feldespáticos y grafitosos fuertemente tectonizados y ausencia de materiales carbonatados. La temperatura media anual en el Albergue Universitario $(2507 \mathrm{~m})$ es de $4,4^{\circ} \mathrm{C}$, con precipitación de $702 \mathrm{~mm} /$ año, de la que el $80 \%$ se concentra entre octubre y abril, mayoritariamente en forma de nieve.

La huella del glaciarismo es visible en el relieve actual y de manera nítida la impronta del Último Máximo Glaciar (Last Glacial Maximum, LGM) (Messerli, 1965, Gómez Ortiz, 1987, Gómez Ortiz et al., 2005) y del Tardiglaciar (Schulte, 2002, Schulte et al., 2002). Asimismo, se ha detectado la Pequeña Edad del Hielo en el seno de los circos (Gómez Ortiz et al., 2006), en los que se estudia el proceso de degradación del hielo fósil glaciar y el permafrost, así como los modelados asociados (Gómez Ortiz et al., 2001, Schulte et al., 2002). 
Desaparecidos los últimos reductos glaciados a mediados de siglo XX, hoy en día el periglaciarismo es patente a partir de los 2500 metros.

Entre las geoformas características del cinturón periglacial cabe destacar los lóbulos de solifluxión, objeto de estudio, emplazados, básicamente, en enclaves de nieves de fusión tardía con vegetación hidrófila, conocidos con el término local de borreguiles (denominados así por su intensiva explotación ganadera).

El área de estudio se centra en las cabeceras de los valles de San Juan y Río Seco. En ellas, antiguos circos glaciares, se concentran un importante número de lóbulos de solifluxión. San Juan es un valle septentrional que acoge más de 150 lóbulos entre los 2 474-2 911 metros. La cobertura vegetal, siempre muy pobre, dispersa y de características herbáceas, ha sido calculada mediante un Sistema de Información Geográfica y resulta en el caso del valle de San Juan en un escaso $3,8 \%$.

En el circo glacial de Río Seco, orientado a mediodía, los borreguiles sólo ocupan un 1,6\% de su superficie, con 46 lóbulos situados entre los 2 900-3 005 metros; casi la totalidad de ellos coinciden en la salida de aguas de las lagunas homónimas del mismo circo. El dispar número de lóbulos de solifluxión en uno $\mathrm{y}$ otro valle se explica por la diferente disponibilidad de agua que se detecta entre vertientes, cuya procedencia está asociada a la mayor duración de la nieve en el suelo.

\section{MATERIALES DE ESTUDIO Y METODOLOGÍA}

La actividad solifluidal actual está condicionada por el régimen hídrico y térmico del suelo. El control del deslizamiento de la capa afectada se ha determinado a partir del seguimiento de estacas de madera (40 cm de longitud) fijadas en el frente y márgenes de diferentes lóbulos. Las estacas se han dispuesto perpendicularmente dentro del lóbulo y fuera de él, en la pendiente, rotulando un trazo continuo en la parte superior de las mismas que ha facilitado el seguimiento centimétrico de estas marcas y el control del movimiento del conjunto lobular a una precisión relativa, suficiente para el objeto de nuestra investigación. Por lo que se refiere a la monitorización de la temperatura del suelo se ha realizado mediante registro térmico continuo (rango 2 horas) almacenado en una cadena de cinco termómetros autónomos y automáticos tipo UTL (Universal Temperature Logger) instalados a las profundidades de 2, 10, 20, 50 y $100 \mathrm{~cm}$ (desde septiembre 2006). Asimismo, el control de la temperatura del aire se llevó a cabo mediante otro dispositivo UTL instalado en la cima del Veleta, a 3398 m, muy cerca del área de estudio.

El estudio sedimentológico de los lóbulos se centró en aquellas unidades previamente sondeadas con una sonda manual de tipo Pürkhauer que evidenciaron una estratigrafía más heterogénea y definida. El análisis de cada muestra se concretó en la granulometría, contenido de materia orgánica $\left(\mathrm{C}_{\mathrm{org}}\right)$, hierro pedogénico $\left(\mathrm{Fe}_{\mathrm{d}}\right)$ y susceptibilidad magnética. Estos trabajos se ejecutaron en el laboratorio de Geografía Física de la Universidad de Berna (Suiza). 


\section{RESULTADOS E INTERPRETACIÓN}

\section{Morfometría y dinamismo}

En los valles de San Juan y Río Seco, se han cartografiado y analizado morfométricamente todos los lóbulos existentes, considerando para cada uno de ellos parámetros tales como: altitud, orientación, pendiente, cobertura vegetal, longitud, anchura, altura del frente y tipología. Esta clasificación se ha adaptado a partir de Hugenholtz y Lewkowicz (2002) y Matsuoka et al., (2005). Entre la variedad de lóbulos identificados en Sierra Nevada, un 72,3\% están vegetalizados y un $27,7 \%$ tienen mayor dominancia de cobertura rocosa. La tabla 1 muestra las principales características morfológicas y morfométricas de los lóbulos en las cabeceras de los valles de San Juan y Río Seco.

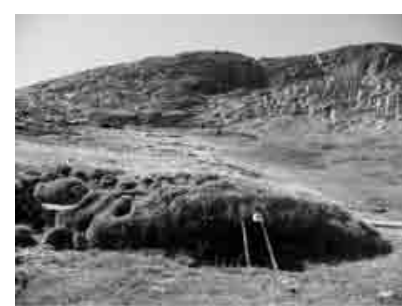

HSL

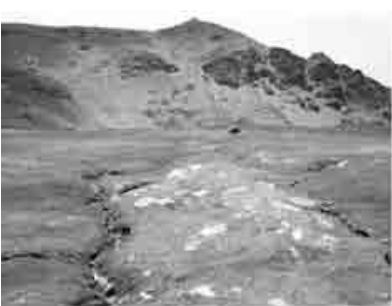

STL-2

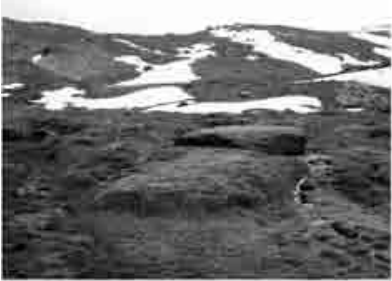

STL-block

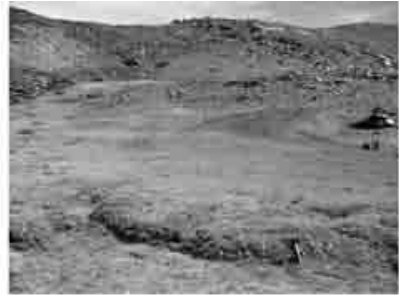

LSL

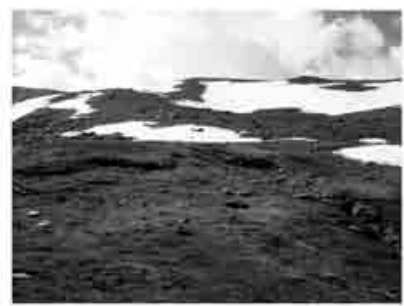

MST

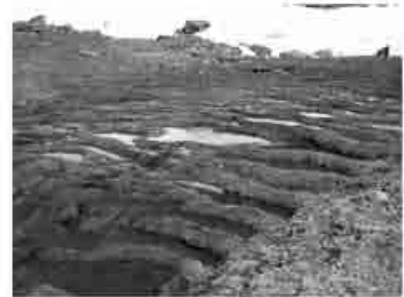

ST

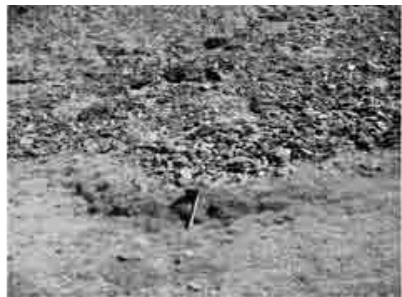

STL-1

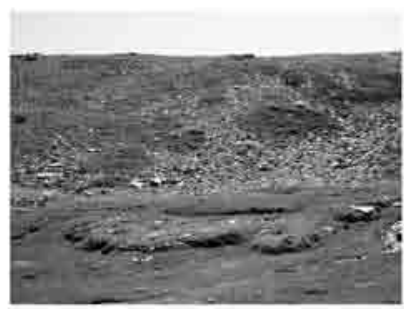

MSS

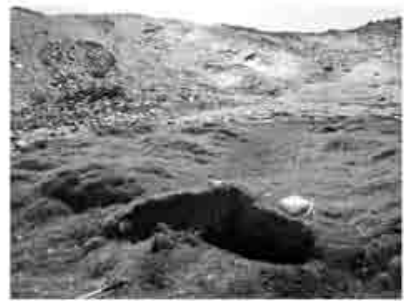

MSL

Fig. 2 - Tipos de lóbulos de solifluxión en Sierra Nevada especificados en la Tabla 1.

Fig. 2 - Types of solifluction landforms in Sierra Nevada listed in Table 1. 
Tabla 1 - Tipología y características de los lóbulos de solifluxión de Sierra Nevada. Table 1 - Typology and associated characteristics of the solifluction landforms in Sierra Nevada.

\begin{tabular}{|c|c|c|c|c|c|c|c|}
\hline Tipo & Características & $\begin{array}{l}\mathrm{N}^{\circ} \text { lóbulos } \\
\text { (control } \\
\text { dinámico) }\end{array}$ & $\begin{array}{l}\text { Activos/ } \\
\text { estables }\end{array}$ & $\begin{array}{c}\text { Lon- } \\
\text { gitud } \\
(\mathrm{m})\end{array}$ & $\begin{array}{l}\text { Ancho } \\
(\mathrm{m})\end{array}$ & $\begin{array}{l}\text { Altura } \\
\text { frente } \\
(\mathrm{m})\end{array}$ & $\begin{array}{l}\text { Pen- } \\
\text { diente } \\
\left({ }^{\circ}\right)\end{array}$ \\
\hline $\begin{array}{l}\text { Low solifluction lobe } \\
\text { LSL }\end{array}$ & $\begin{array}{l}\text { Predomimancia } \\
\text { de turba con un } \\
\text { frente }<80 \mathrm{~cm}\end{array}$ & $\begin{array}{l}104 \\
(4)\end{array}$ & $2 / 2$ & 4,4 & 3,6 & 0,47 & 9,8 \\
\hline $\begin{array}{l}\text { High solifluction lobe } \\
\text { HSL }\end{array}$ & $\begin{array}{l}\text { Predomimancia } \\
\text { de turba con un } \\
\text { frente } \geq 80 \mathrm{~cm}\end{array}$ & $\begin{array}{l}32 \\
(5)\end{array}$ & $1 / 4$ & 6,6 & 5,3 & 0,9 & 11,5 \\
\hline $\begin{array}{l}\text { Stone-banked lobe-1 } \\
\text { STL-1 }\end{array}$ & $\begin{array}{l}\text { Abundancia de } \\
\text { gravas/rocas } \\
>50 \%\end{array}$ & $\begin{array}{c}6 \\
(1)\end{array}$ & $1 / 0$ & 5,1 & 3,4 & 0,39 & 13,0 \\
\hline $\begin{array}{l}\text { Stone-banked lobe-2 } \\
\text { STL-2 }\end{array}$ & $\begin{array}{l}\text { Abundancia } \\
\text { de gravas/rocas } \\
<50 \%\end{array}$ & $\begin{array}{l}34 \\
(2)\end{array}$ & $1 / 1$ & 5,4 & 4,5 & 0,52 & 10,3 \\
\hline $\begin{array}{l}\text { Turf-mantled lobes } \\
\text { MST }\end{array}$ & $\begin{array}{l}\text { Manto de } \\
\text { solifluxión con } \\
\text { predominancia } \\
\text { de turba } \geq 8 \mathrm{~m}\end{array}$ & $\begin{array}{c}6 \\
(1)\end{array}$ & $0 / 1$ & 13,3 & 12,7 & 0,78 & 13,1 \\
\hline $\begin{array}{l}\text { Stone-mantled lobes } \\
\text { MSS }\end{array}$ & $\begin{array}{l}\text { Manto de } \\
\text { solifluxión con } \\
\text { predominancia } \\
\text { de gravas/rocas } \\
\geq 8 \mathrm{~m}\end{array}$ & $\begin{array}{l}11 \\
(0)\end{array}$ & $0 / 0$ & 9,9 & 9,5 & 0,86 & 14,0 \\
\hline $\begin{array}{l}\text { Ploughing boulders } \\
\text { STL (block) }\end{array}$ & Bloque arador & $\begin{array}{c}4 \\
(1)\end{array}$ & $1 / 0$ & 5,0 & 2,8 & 0,67 & 8,0 \\
\hline $\begin{array}{l}\text { Solifluction terrassettes } \\
\text { ST }\end{array}$ & $\begin{array}{l}\text { Pequeñas } \\
\text { terrazas de } \\
\text { solifluxión }\end{array}$ & $\begin{array}{c}3 \\
(2)\end{array}$ & $0 / 1$ & 1,1 & 0,8 & 0,24 & 10,0 \\
\hline $\begin{array}{l}\text { Mudflow-affected } \\
\text { solifluction lobes } \\
\text { MSL }\end{array}$ & $\begin{array}{l}\text { Lóbulos de } \\
\text { forma irregular } \\
\text { con matriz } \\
\text { fangosa }\end{array}$ & $\begin{array}{c}2 \\
(1)\end{array}$ & $0 / 1$ & 5,5 & 7,5 & 0,67 & 8,5 \\
\hline
\end{tabular}

Los datos del periodo agosto 2006 - agosto 2007 reflejan inactividad de movimiento solifluidal. Únicamente escasos desplazamientos en puntos muy concretos y particulares se han detectado. Así sucede en los lóbulos cercanos a los neveros más tardíos o en aquellos más próximos a los cursos fluviales en los que se registran tasas de desplazamiento, aunque en todos los casos inferiores a 0,5 $\mathrm{cm} /$ año. El grado de disponibilidad de agua es el factor más relevante que determina la solifluxión en Sierra Nevada. Finales de primavera e inicios de verano parece el momento propicio para que se active la solifluxión en el macizo, cuando la infiltración de agua procedente de la fusión nival en presencia de suelo profundo 
aún helado puede facilitar el deslizamiento de la parte superficial de suelo. El recubrimiento vegetal en los lóbulos tiende a actuar como elemento limitante a la solifluxión, siendo los lóbulos no vegetalizados los que muestran tasas más elevadas de desplazamiento. A su vez, la pendiente juega un papel clave: vertientes más inclinadas favorecen una escorrentía más rápida (con una menor permanencia del elemento blanco en el suelo) que dificulta el crecimiento vegetal, favoreciendo así la movilización mineral y los movimientos de vertiente.

\section{Control térmico}

En uno de los lóbulos de solifluxión del circo del Río Seco (3 $001 \mathrm{~m}$ ) se instalaron un total de 5 sensores térmicos UTL a las profundidades de 2, 10, 20, 50 y $100 \mathrm{~cm}$. Los registros térmicos de septiembre de 2006 a agosto de 2007 muestran inexistencia de permafrost aunque cabe destacar la presencia de una profunda capa helada estacional que se alarga desde noviembre de 2006 hasta junio de 2007. La capa helada de $70 \mathrm{~cm}$ de profundidad, empezó a formarse a principios de noviembre con las primeras temperaturas negativas. La inestabilidad del manto nival en el suelo durante el inicio del invierno se tradujo en una elevada sensibilidad de los sensores térmicos más superficiales con bruscos aumentos y descensos de temperatura. La estructura interna del lóbulo, con ligeras variaciones granulométricas en su perfil (cambios en el porcentaje de arenas de entre 40-65\%), parece no influir en la penetración de la onda térmica en el subsuelo. Una primavera relativamente fría y nivosa con temperaturas
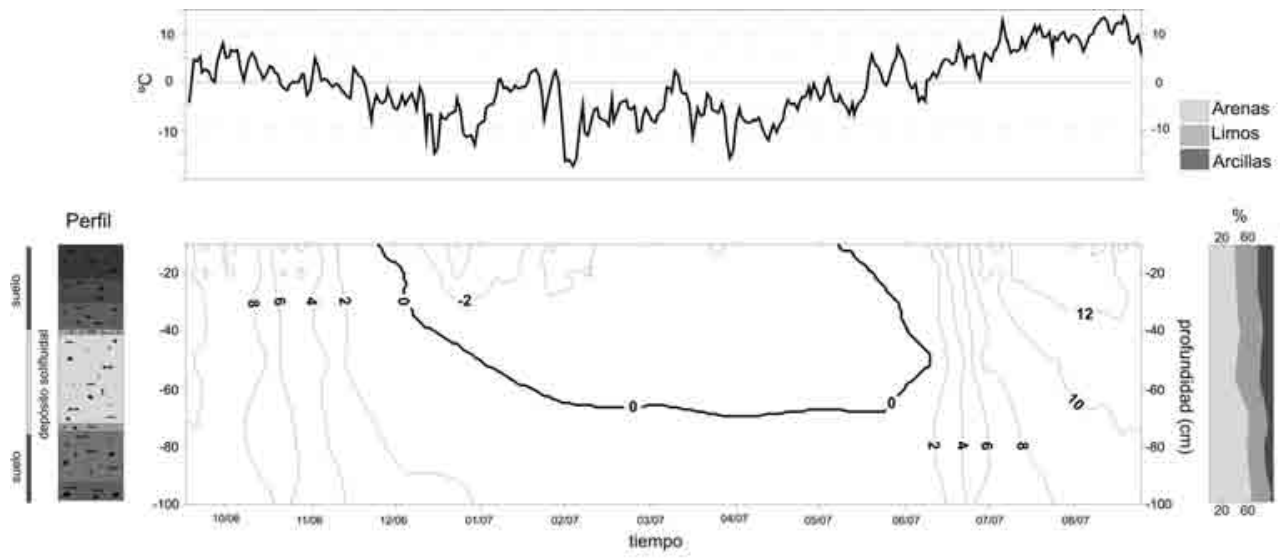

Fig. 3 - Temperatura media diaria del aire en el Picacho del Veleta, $3398 \mathrm{~m}$ (figura superior) y evolución térmica del suelo en un lóbulo del circo de Rio Seco (figura inferior), desde setiembre del 2006 hasta agosto del 2007 con su correspondiente perfil y fracción fina.

Fig. 3 - Mean daily air temperature at the Veleta peak, $3398 \mathrm{~m}$ (upper image), and evolution of the ground temperature in a solifluction lobe of the Rio Seco cirque (lower image), from

September 2006 to August 2007, plotted with its profile and grain-size distribution. 
claramente negativas conservó el manto nival hasta finales de mayo, aunque los valores térmicos de hasta 7 y $8^{\circ} \mathrm{C}$ de temperatura media diaria de principio de junio propiciaron la fusión acelerada de la protección nival y el rápido deshielo del lóbulo. Así pues, a pesar de la existencia de un horizonte helado de más de $70 \mathrm{~cm}$ y que perduró 8 meses, no se dieron las condiciones climáticas propicias para activar la solifluxión en este lóbulo durante el periodo de estudio.

\section{Registros solifluidales durante el Holoceno tardío}

El estudio de la estructura interna de numerosos lóbulos de solifluxión pone de manifiesto la sucesión de fases solifluidales y procesos edáficos entre los 2 500-3 000 metros en Sierra Nevada durante los últimos milenios. La figura 4 muestra la litoestratigrafía y la analítica geoquímica del perfil del lóbulo RS3 del circo de Río Seco, datado radiométricamente por Esteban (1994).

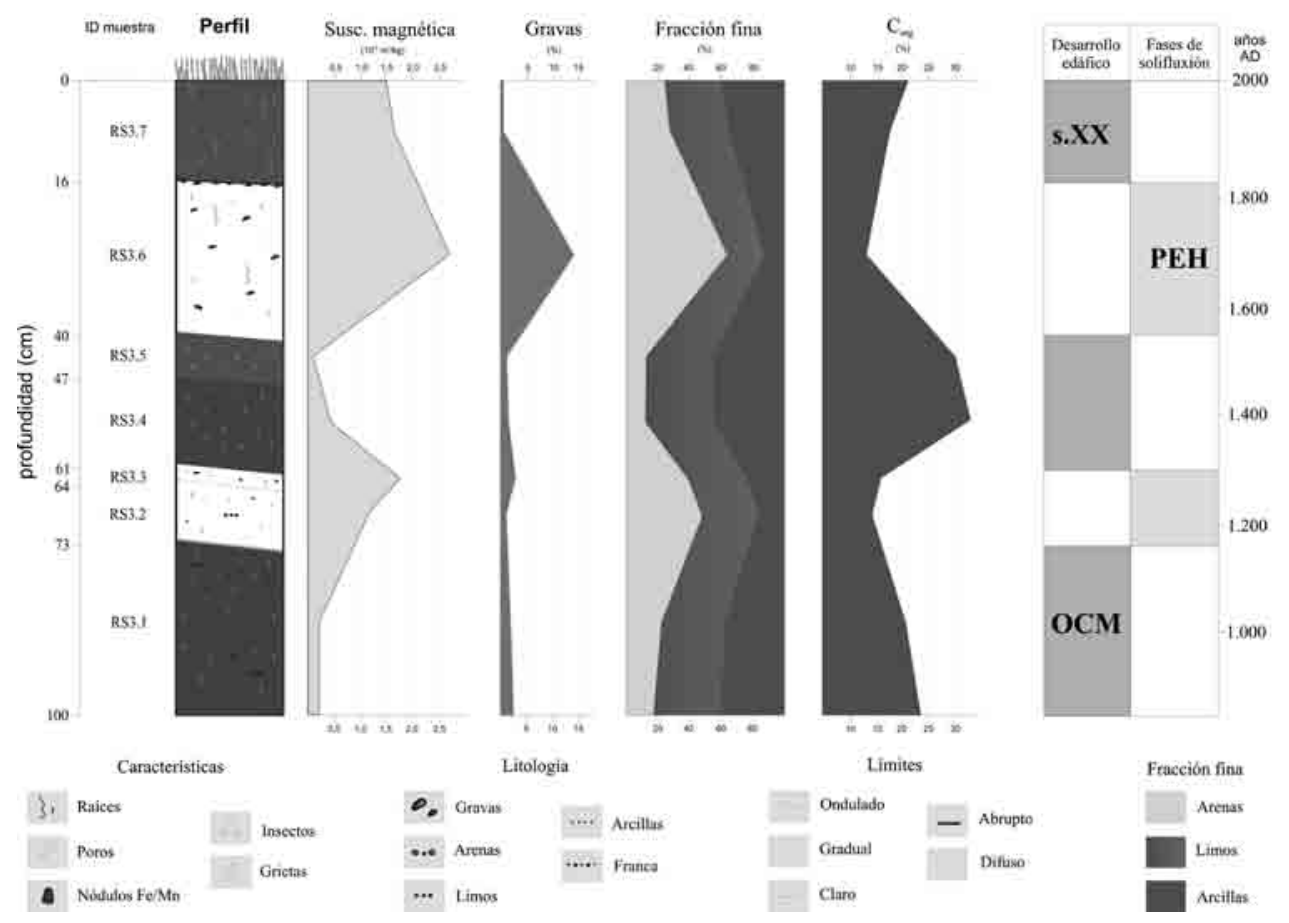

Fig. 4 - Litoestratigrafía de un lóbulo de solifluxión en Río Seco.

Fig. 4 - Lithostratigraphy of a solifluction lobe in Rio Seco. 
La base del lóbulo corresponde al Óptimo Climático Medieval (OCM), fase cálida que en este sector de Río Seco se refleja en la formación de suelos, con elevados contenidos de materia orgánica, $\mathrm{Fe}_{\mathrm{d}} \mathrm{y}$ material fino. El desarrollo edáfico se vio truncado con el deterioro climático acontecido durante la Pequeña Edad del Hielo (PEH), evidenciándose la inestabilidad de vertientes con dos fases solifluidales con abundancia de gravas y arenas y disminución de la materia orgánica. Los dos empujes solifluidales que se detectan parecen corresponderse con las dos pulsaciones frías indicadas por Schulte (2002), a partir de dataciones de ${ }^{210} \mathrm{~Pb}$ en el cercano Corral del Veleta. La parte orgánica superior del perfil podría resultar del aumento térmico registrado desde mediados de siglo XIX que fosiliza el depósito solifluidal superior de la PEH.

Durante esta fase fría, la mayor presencia de manchas de nieve tardías y neveros como evidencian las fuentes documentales (Rodrigo, 1994) y los escritos de época (Gómez Ortiz y Plana Castellví 2006), supondrían un suministro líquido más duradero alargando así el periodo de potencial actividad solifluidal. Los registros sedimentarios evidencian que fases frías y/o húmedas favorecen la solifluxión e inestabilizan las laderas, mientras que periodos más cálidos inducen al desarrollo del tapiz vegetal posiblemente debido a un mayor período vegetativo, formación de suelos y estabilización de vertientes

\section{CONCLUSIONES}

Los resultados del control térmico y la monitorización en lóbulos de solifluxión de Sierra Nevada muestran su estabilidad, muy condicionada por el régimen climático imperante en el macizo. La fusión de un horizonte helado de hasta $70 \mathrm{~cm}$ de profundidad, que se mantiene desde noviembre hasta principios de junio, no genera desplazamiento solifluidal. En cuanto a la génesis de los lóbulos hay que señalar que los perfiles sedimentológicos de muchos de ellos constatan la existencia de fases más frías y/o húmedas durante el Holoceno tardío que permitieron activar la solifluxión mientras que la presencia de episodios cálidos, como el actual, tendieron a favorecer el desarrollo de suelos incipientes. A diferencia de las previsiones de Lewkowickz y Clarke (1998) para ámbitos de altas latitudes con permafrost, que proponen que la actual inercia al calentamiento global reactivará e intensificará la solifluxión, en Sierra Nevada, probablemente y a manera de hipótesis de trabajo para los actuales enclaves de borreguiles, esta tendencia podría propiciar el desarrollo de suelos.

\section{AGRADECIMIENTOS}

La presente investigación se enmarca en el programa de Formación de Personal Universitario (FPU) del Ministerio de Educación y Ciencia. También el agradecimiento a los proyectos 017/2007 de Parques Nacionales (MMA) y a Fluvalps-3000 (CGL2006-01111). 
Igualmente, cabe agradecer la disponibilidad del Dr. Heinz Veit de la Universidad de Berna, que nos permitió realizar el trabajo de laboratorio en este centro suizo y las sugerencias de los revisores anónimos que perfilaran el redactado final del presente articulo.

\section{BIBLIOGRAFÍA}

Ballantyne C K, Harris C (1994) The Periglaciation of Great Britain. Cambridge Univ. Press, Cambridge.

Chueca J, Julián A (1995) Cuantificaclón de movimientos en masa lentos en medios de montaña: Pirineo Central. Lurralde 18: 173-196.

Esteban A (1994) Evolución del paisaje durante los últimos 10.000 años en las montañas del Mediterráneo occidental: ejemplos del Pirineo Oriental y Sierra Nevada. Tesis doctoral. Universidad de Barcelona.

Gómez Ortiz A (1987) Morfología glaciar en la vertiente meridional de Sierra Nevada (Área VeletaMulhacén). Estudios Geográficos, 193: 527-558.

Gómez Ortiz A, Plana Castellví J A (2006) La Pequeña Edad del Hielo en Sierra Nevada a partir de los escritos de la época (siglos XVIII y XIX) y relaciones con el progreso de la geografía física y geomorfología españolas. Boletín de la Asociación de Geógrafos Españoles, 42: 71-98

Gómez Ortiz A, Palacios Estremera D, Luengo Nicolau E, Tanarro García L M, Schulte L, Ramos Sainz M, Salvador Franch F (2002) Inestabilidad de taludes y cubierta nival en áreas marginales de permafrost. El caso de la pared norte del picacho del Veleta (Sierra Nevada. España). Revista de Geografia, 1: 35-56.

Gómez Ortiz A, Palacios D, Ramos M, Tanarro L M, Salvador Franch F (2001) Location of permafrost in marginal regions: Corral Del Veleta, Sierra Nevada, Spain. Permafrost Periglac. Process. 12(1): 92-110.

Gómez Ortiz A, Schulte L, Salvador F, Palacios D, Sanz de Galdeano C, Sanjosé J J, Tanarro L M, Atkinson A (2005) Field trip to Sierra Nevada Massif Glacial Geomorphology and Present Cold Processes. 6th International Conference on Geomorphology. September 7-11, Zaragoza (Spain).

Hugenholtz C H, Lewkowicz A G (2002) Morphometry and environmental characteristics of turfbanked solifluction lobes, Kluane Range, Yukon Territory, Canada. Permafrost Periglac. Process. 13: 301-313.

Lewkowicz A G, Clarke S (1998) Late-summer solifluction and active layer depths, Forsheim Peninsula, Ellesmere Island, Canada. In Lewkowicz, A.G. y Allard, M. (eds), Proceedings of the Sixth International Conference on Permafrost, Yellowknife, Université Laval, Sainte-Foy: 641-666.

Matsuoka N, Ikeda A, Date T (2005) Morphometric analysis of solifluction lobes and rock glaciers in the Swiss Alps. Permafrost Periglac. Process, 16: 99-113.

Messerli B (1965) Beiträge zur geomorphologie der Sierra Nevada (Andalusien). Juris Verlag. Zürich.

Molero Mesa J, Pérez Raya F (1987) La flora de Sierra Nevada. Granada, Spain. University of Granada.

Palacios D, Andrés N de, Luengo E (2003) Distribution and effectiveness of nivation in Mediterranean mountains: Peñalara (Spain). Geomorphology 54: 157-178. 
Rodrigo F (1994) Cambio climático natural. La Pequeña Edad de Hielo en Andalucía. Tesis doctoral, Universidad de Granada.

Schulte L (2002) Climatic and human influence on river systems and glacier fluctuations in southeast Spain. Quaternary International, 93-94: 85-100.

Schulte L, Marcos Garcia-Blanco J, Gómez Ortiz A, Palacios Estrema D, Tanarro Garcia M, Fernández Fernández A, Ramos Sainz M (2002) Evolución glaciar y periglaciar del Circo del Mulhacén (Sierra Nevada, Península Ibérica). Publicaciones del IGME. Serie Geología, 1: 491-499.

Vieira G (2004) Geomorfologia dos planaltos e altos vales da Serra da Estrela. Ambientes frios do Plistocénico Superior e dinâmica actual. Tese de doutoramento, Universidade de Lisboa.

Wanner H, Beck C, Brádzil R, Casty C, Deutsch M, Glaser R, Jacobeit J, Luterbacher J, Pfister C, Pohl S, Sturm K, Werner P C, Xoplaki E (2004) Dynamic and socioeconomic aspects of historical floods in central Europe. Erdkunde 58: 1-16. 